Revue internationale P.M.E.

Économie et gestion de la petite et moyenne entreprise

\title{
Structures sociales et aspects institutionnels des systèmes d'industrialisation diffuse de l'Italie du Centre
}

\section{Angelo Michelsons et Clerici Vagantes}

Volume 2, numéro 2-3, 1989

URI : https://id.erudit.org/iderudit/1007934ar

DOI : https://doi.org/10.7202/1007934ar

Aller au sommaire du numéro

Éditeur(s)

Presses de l'Université du Québec

ISSN

0776-5436 (imprimé)

1918-9699 (numérique)

Découvrir la revue

Citer cet article

Michelsons, A. \& Vagantes, C. (1989). Structures sociales et aspects institutionnels des systèmes d'industrialisation diffuse de l'Italie du Centre. Revue internationale P.M.E., 2(2-3), 185-200. https://doi.org/10.7202/1007934ar
Résumé de l'article

Les systèmes à «industrialisation diffuse», qui apparaissaient dans les régions du Centre et du Nord-est de l'Italie au cours des années soixante et soixante-dix, ne peuvent être expliqués exclusivement à l'aide d'instruments analytiques économiques, compte tenu du rôle central des relations sodales et institutionnelles en ce qui concerne l'organisation, le fonctionnement et la régulation de l'économie. Cet article retrace rapidement les traits particuliers de ce qu'on a pris l'habitude d'appeler «le modèle Italien». 


\title{
Structures sociales et aspects institutionnels des systèmes d'industrialisation diffuse de l'Italie du Centre
}

\author{
Angelo MICHELSONS \\ Clerici Vagantes, Turin
}

\begin{abstract}
RÉSUME
Les systèmes à «industrialisation diffuse", qui apparaissaient dans les régions du Centre et du Nord-est de l'Italie au cours des années soixante et soixante-dix, ne peuvent être expliqués exclusivement à l'aide d'instruments analytiques économiques, compte tenu du rôle central des relations sociales et institutionnelles en ce qui concerne l'organisation, le fonctionnement et la régulation de l'économie. Cet article retrace rapidement les traits particuliers de ce qu'on a pris I'habitude d'appeler "le modèle Italien".
\end{abstract}

\section{ABSTRACT}

The "diffused industrialisation" systems which emerged in the central sud north-east regions of Italy during the 1960 s and 1970s cannot be explained by using the tools of economics only, given the big role played in those regions by social and institutional relations in the organisation, working and regulation of the economy. The paper sketches out a brief outline of these features of the so-called a Italian model'".

\section{RESUMEN}

Los sistemas de industrializacion difusa, que aparecen en las regiones del Centro y del Noreste de Italia en el transcurso de los anos sesenta y setenta, no pueden ser explicados exclusivamente con la ayuda de los instrumentos analiticos economicos, a causa del papel central de las relaciones sociales e institucionales en cuanto a la organizacion, el funcionamiento y la regulacion de la economia. Este articulo describe rapidamente los razgos particulares de lo que acostumbramos llamor «El Modelo Italiano".

- Angelo Michelsons, $\mathrm{Ph}$. D. en sociologie économique à Cambridge (Grande-Bretagne) a mené plusieurs recherches sur les structures productives de Turin (Fiat), de la zone du Canavese au Piemont (Olivetti) et de la Toscane (PME). Dès 1988, il gère la société de recherche sociale «Clerici Vagantes» de Turin (Italie). Adresse: Clerici Vagantes Corso Moncalleri, 23/0 - 10100 Torino. 


\section{Les règles de coopération entre l'économie et la société}

L'émergence et le succès durant les années 70 d'un autre modèle d'organisation industrielle, différent de la grande entreprise engagée dans la production de masses, modèle basé sur un système intégré de PMI et sur les interactions entre l'économie et la société, a requis des observateurs de remarquables innovations sur le plan théorique. Premièrement, ils ont dû reconnaître l'existence d'un cheminement alternatif de développement et des nouvelles solutions pour soutenir la croissance économique; deuxièmement, ils ont dû admettre l'insuffisance d'une explication purement économique pour ce phénomène d'espaces industriels diffus ${ }^{1}$, et la nécessité d'accorder pour cela autant d'attention aux dynamiques sociales et aux aménagements institutionnels.

Du point de vue économique, la petite dimension peut ainsi constituer la base permettant de développer avant tout une stratégie particulière réunissant les conditions suivantes : une production comprenant différentes phases pouvant techniquement exister séparément les unes des autres, et une demande diversifiée variant avec le temps (Brusco, 1975; Becattini, 1989). La séparation technique des diverses phases de production augmente au cours des années, à la suite de changements dans la technologie énergétique, des systèmes d'information ou des réseaux de transport; cette demande variable et diversifiée est caractéristique de nombreux secteurs traditionnels à moyenne et basse technologie comme les chaussures, le cuir, le meuble, la bonneterie, la céramique, mais aussi de secteurs plus avancés comme les produits d'équipements et d'automatisation industrielle. Ces conditions se sont développées en Italie durant les années 50 à partir de systèmes de petites et moyennes entreprises et de la tendance à la spécialisation territoriale.

Chaque entreprise du système n'a qu'une importance relative alors que le système entier peut acquérir un poids économique certain. Dans les systèmes plus traditionnels, la majeure partie des unités productives sont à peu près de même taille et sont directement en concurrence; en général, on peut y trouver une division du travail entre les entreprises qui constituent la base technique pour l'instauration des rapports des coopérations entre elles. Comme l'ont montré de nombreuses études

1. Nombreuses sont les définitions des systèmes locaux de petite entreprise - district industriel, espace-système, modèle Nec, etc. - élaborées par différents chercheurs et scientifiques sociaux durant les dernières années. Cette variété de définitions reflètent différentes approches théoriques et porte sur différents cas concernant ces questions. L'élément commun à ces différents cas est stratégiquement relié au fonctionnement du modèle, à l'existence d'une organisation à orientation sociale du marché et, par conséquent, à la tendance diffuse à la formation d'un entrepreneuriat autonome. Néanmoins, dans ce contexte, j'utilise la définition «espace d'industrialisation diffuse», orginalement élaborée par Bagnasco, qui résume les différentes typologies des systèmes de petites entreprises. 
d'économie industrielle, il faut bien comprendre que tant cette concurrence que cette coopération entre les entreprises dans le système territorial constituent sa «force». La concurrence est un élément de base parce que c'est à travers le marché que se vérifie la validité de la technique productive choisie et le champ d'opération de chaque entreprise. Mais la coopération représente une fonction encore plus importante, parce qu'elle explique la redistribution des commandes, la circulation des connaissances techniques, l'accession facile au crédit, etc. Cette coopération entre les petites et moyennes entreprises d'un système territorial ne peut cependant pas être interprétée comme une simple application d'une technique organisée, comme un choix des agents économiques qui, à la suite d'un calcul avantages/coûts auraient convenu d'organiser l'économie sur un tel modèle. Plutôt, cette coopération doit être vue comme le produit historique des relations de confiance instaurées au niveau des communautés locales (Michelson, 1989).

Ces considérations permettent d'expliquer le fonctionnement économique des espaces d'industrialisation diffuse comme un ensemble de rapports sociaux en tant que ressources structurelles permettant d'expliquer l'origine et le comportement particulier de chaque district.

Plus forte est cette coopération, plus les rapports de marché et les relations informelles de la communauté peuvent permettre de faire face à l'instabilité traditionnelle du cycle économique et de profiter des possibilités d'accumulations technologiques et de la croissance du bien-être de la communauté locale. Au contraire, plus faible est celle-ci moins la coopération entre les entreprises et plus les exigences de la concurrence sont reconfirmées, plus chaque firme s'orientera vers une stratégie individuelle et moins cette conception du système productif agira à la base de leur développement comme ressource pour la communauté locale. Par exemple, un simple calcul économique poussera chaque entreprise à internationaliser ses phases productives, à se diriger vers des fournisseurs externes, ou encore à se diriger vers des productions plus standards qui demandent une échelle de production plus grande. Ou bien, en phase descendante du cycle économique, la non-coopération l'amènera à abaisser le salaire des ouvriers, à rechercher de nouvelles solutions techniques et de nouveaux produits, en contradiction avec les règles admises des institutions locales.

Ce problème a déjà été étudié en références aux districts industriels flexibles du siècle passé. Dans ces districts, on a retrouvé des caractéristiques analogues à ce qu'on connait aujourd'hui, soient des institutions permettant de favoriser cet équilibre entre coopération et concurrence entre les entreprises. Ces institutions créaient ainsi «un environnement dans lequel les investissements en capitaux et en habiletés pourraient être constamment recombinés de façon à produire rapidement de nouveaux assortiments de biens et, comme précondition à ceci, une situation décourageant les firmes de se compétitionner en réduisant les salaires et en coupant les prix plutôt qu'en creant de nouveaux produits et de nouveaux processus de production» (Sabel et Zeitlin, 1985: 144). Un tel encadrement des entreprises locales se rencontre 
encore dans les espaces ou les districts contemporains. Par exemple, dans le district de l'industrie du meuble de la Haute-Valdesa, la forte récession du début des années 1980 a accéléré les processus de restructuration de chaque entreprise, a entraîné une riposte rapide institutionnelle de la part des syndicats, des entrepreneurs et des politiciens, a favorisé le financement pour l'acquisition et la diffusion des nouvelles technologies et des nouvelles habiletés dans le district et a causé une nouvelle consolidation de la coopération inter-entreprise (Baptiste et Michelsons, 1988). Actuellement, comme dans le passé, cette limitation de la concurrence aux aspects de l'innovation a favorisé l'efficience et la compétition du système économique dans son ensemble, et entraîné le renouvellement des ressources par la communauté locale; ceci a eu pour effet de renforcer l'identité et la médiation des intérêts de tous.

Le cadre institutionnel qui rend possible la flexibilité et la recherche de l'innovation varie selon le niveau technologique, les caractéristiques économiques et l'histoire sociale de l'espace industriel. Sur l'aspect historique des districts, «analytiquement, il est possible de distinguer trois systèmes : l'un basé sur la municipalité, un autre sur le comportement paternaliste ou d'Etat-providence des compagnies capitalistes, un troisième sur les firmes fonctionnant en coopératives (...). Mais une industrie peut évoluer d'un système à l'autre (...) et il y a plusieurs cas hybrides où les institutions ont combiné les traits de deux de ces systèmes de base» (Sabel etZeitlin, 1985: 147). Actuellement, les connaissances sont encore trop fragmentaires pour permettre une modélisation analogue : de changements en changements, les situations évoluent et présentent une réalité institutionnelle différente du passé, comme par exemple dans le cas de Olivetti (Michelsons, 1989). Toutefois, la fonction fondamentale d'un tel système institutionnel semble rester valide pour promouvoir l'innovation, l'efficience et la flexibilité du système productif, au profit de la communauté locale.

Les pages suivantes traiteront des principales caractéristiques sociales et industrielles des districts connus de la «troisième Italie» ou de «l'Italie du Centre». Ceux-ci, sont désormais formalisés dans un modèle général. Il faut cependant dire que ce modèle stratégique fonctionne, somme toute, essentiellement de façon heuristique : le système dynamique des petites entreprises requiert, pour cela, des solutions institutionnelles à orientations sociales qui répondent aux problèmes régionaux du système; mais ces orientations n'impliquent pas nécessairement les mêmes réponses «spontanées» dans chaque région, industrie ou époque provenant d'un processus historique complexe. 


\section{Le processus d'industrialisation}

Le processus d'industrialisation diffuse qui fonctionne dans plusieurs zones des régions italiennescentrales et nord-orientales(Emilie-Romanie, Toscane, Umbrie, Marche, Veneto, Trentino) a généralement commencé dans les années suivant le dernier conflit mondial. Cela ne signifie pas que ces régions étaient privées d'industries traditionnelles, artisanales et commerciales. Au contraire, les phénomènes comme le système de production domestique («putting-out») et la proto-industrie étaient en grande partie des caractéristiques de ces zones; dans plusieurs de ces villes de ces régions on pouvait retrouver tant une tradition de production spécialisée et de haute qualité pour les marchés internationnaux que des restes du bas moyen-age. En outre, la première vague de l'industrialisation italienne a porté sur la localisation de nombreux grands établissements dans les grandes villes, à côté de zones de productions artisanales. Toutefois, à la fin de la guerre le centre-nord-est est encore un territoire à prédominance agricole. Alors que la politique nationale privilégie la concentration des grands investissements dans le «triangle industriel» du nord-ouest (grosso modo, la zone comprise entre Milan, Turin et Genève). Cela conduit en peu d'années à une forte immigration du sud et des urbanisations chaotiques.

Le désintéressement de l'Etat pour le développement économique de ces autres régions, qui se poursuit aussi durant les décennies successives jusqu'à ce que la politique nationale encourage des investissements moyens à base de grands établissements dans les régions méridionales sous-développées, n'empêche pas un processus original d'industrialisation dans ces régions. Structurellement, les caractéristiques de départ communes à ces régions peuvent se résumer d'abord au manque de prolétarisation dans l'agriculture et à la prédominance de petites unités productives exploitées par les métayers, les fermiers ou les exploitants, ensuite à l'absence d'investissements importants sinon à des désinvestissements dans des établissements industriels de moyennes tailles, et enfin à un marché diffus.

Premièrement, la dispersion de la population à la campagne, la prévalence de rapports de production relativement autonome et basé sur le métayage et la location de la petite propriété villageoise, semble constituer un des éléments fondamentaux de la matrice sociale à l'origine de cette société à industrialisation diffuse. Il est ainsi «possible d'établir une corrélation statistique significative», au niveau de la province entre la présence de plus grands et de plus petits rapports de production autonome (en agriculture) à partir de 1901 (le recensement) et de plus ou moins grande présence de petites firmes après la (seconde) guerre (Bagnasco, 1985 : 124). Deuxièmement, pour une interprétation plus diffuse, la prépondérence des rapports de production autonome dans les campagnes a fourni des ressources fondamentales au développement des petites entreprises. Comme on le verra dans la prochaine section, ces ressources relèvent en particulier d'une offre d'emploi peu coûteuse et flexible, d'une source pour la création des capitaux risqués (par l'entremise des épargnes familiales) et d'un lieu de formation des aptitudes entrepreneuriales ou pré-entrepreneuriales. Cette corrélation statistique doit toutefois être considé- 
rée avec une certaine prudence. Elle semble valable pour l'Italie du Centre; elle doit être cependant vue comme une pré-condition nécessaire mais non suffisante à la mise en marche d'un processus d'industrialisation diffuse.

Avant tout, il faut reconnaître que de nombreux cas connus de districts industriels ont pour origine une matrice sociale fort différente. Par exemple, il existe des districts en milieu urbain (du textile à New-York à l'automatisation industrielle à Turin : Piore et Sabel, 1984; Bianco et Luciano, 1982), ou des districts développés pour répondre aux besoins spécifiques des grandes entreprises, ou encore des cas encore plus particuliers comme le district de la chaussure créé au Brésil dans une communauté d'artisans spécialisés provenant de Amburgo (Sabel, 1986). En outre, il n'existe pas beaucoup de cas où une grande entreprise a tiré avantages, en terme d'offres de travailleurs, d'une localisation dans une zone rurale où prédominaient des rapports de production autonome; de tels modèles de localisation se retrouvent, par exemple, en France (Saglio, 1989), mais aussi dans d'autres régions italiennes (Scamuzzi, 1987). Sans soutenir que l'industrialisation diffuse peut constituer une solution aux problèmes considérés dans des modèles plus traditionnels basés sur la localisation d'une grande entreprise dans une zone rurale, ces considérations soulignent l'importance d'autres éléments structuraux discutés au début de cette section.

En effet, ces différentes situations «de gaspillage d'investissements» ont touché, après la seconde guerre, une grande partie des régions de l'Italie du Centre. Des capitaux accumulés en agriculture dans ces régions, seule une partie a été investie dans les activités industrielles, et très souvent, à l'extérieur même des régions. Ce phénomène souligné en particulier dans le cas de l'Emilie-Romagne (Capecchi, 1987) et de la Toscane (Becattini, 1975), doit avoir quelques liaisons décidément politiques. La fermeture d'établissements de production de guerre, la non-modernisation des autres établissements et le manque d'affluence de nouveaux capitaux foumissent une explication - encore qu'elle n'est pas l'unique - reprise par les gouvernements locaux de gauche, à prédominance communiste, dans les deux régions. Le gaspillage de ces investissements et les démantellements ont «libéré» un nombre assez élevé d'ouvriers qualifiés. Ceux-ci ont deux choix possibles : ou rejoindre le flux des émigrants vers les grandes villes du Nord ou chercher de nouvelles voies de travail autonome dans la région (Capecchi, $1987: 253$ ),

Les opérateurs et travailleurs agricolès de ces régions avaient le choix de ne pas émigrer. Cette «mobilisation du marché» (Bagnasco, 1988) n'était pas dérivée d'un processus planifié. Même si elle était fondée sur une large diffusion de stratégies économiques acquises, elle a permis d'orienter l'épargne pour le réinvestissement et l'acceptation du marché comme régulateur de récompenses et des hiérarchies sociales, librement définies et pour cela même acceptées.

Cette culture de contrats et de marchés à la base des valeurs et des orientations déjà présentes et socialisées dans la famille n'explique toutefois pas les comportements individuels, mais s'est plutôt concrétisée dans un projet collectif. Comme le rappellent Becattini $(1975,1978)$ et Capecchi $(1985,1987$, et al. 1988) 
l'analyse des entretiens d'artisans de la génération des années 1950-1970 permet de dégager une conception du passage d'ouvrier à artisan comme acte d'opposition aux capitalistes de la grande entreprise, comme un parcours collectif qui ne modifie pas l'appartenance de classe (Capecchi, 1987: 258). De plus, souvent les fondements de ce projet collectif ont été jetésà travers une coalition entre les luttes ouvrières et celles des métayers de l'immédiate après-guerre (Beccatini, 1975). L'identification d'un «ennemi» commun qui refusait d'investir pour offrir de nouveaux postes pour les travailleurs industriels et pour améliorer les conditions de vie des métayers, et de soutenir un développement accessible à l'un et l'autre de ces classes sociales à fournir une cause majeure pour favoriser la coopération et l'identification à la communauté de ces sociétés à industrialisation diffuse (Baptiste et Michelsons, 1988).

Cette dimension du «projet collectif» tiré de cette identification typique dans ce modèle de développement, $n$ 'a pas eu le même succès dans les zones à prévalence démocrate-chrétienne (les régions de l'Italie du centre-oriental). Comme on le verra à l'avant dernière section, dans des régions, les projets collectifs de développement basés sur une «défense collective» (Trigilia, 1986) ont eu plutôt des effets plus négatifs encouragés par l'industrialisation nationale.

\section{Les ressources de base : le travail}

La brève description économique des systèmes des petites entreprises (vue à la première section) ne touchait pas au problème du prix et de l'élasticité du travail. Ces éléments relèvent individuellement des conditions de l'économie et de l'organisation reliées aux avantages de la petite dimension, quelque soit le coût du travail. Toutefois, il faut se rappeler qu'un coût moindre et une faible rigidité dans l'utilisation de la force de travail ont représenté une ressource importante et souvent décisive, pour les entreprises des secteurs plus traditionnels et à technologie plus simple, et dans les phases de «décollage» de plusieurs zones industrielles de l'Italie du Centre.

Ces caractéristiques de l'offre du travail doivent être strictement jointes à la structure des rapports de production en agriculture et au rôle de la famille. La famille constitue avant tout la pierre angulaire de cette société. Mais on parle ici d'un type particulier de la famille paysanne élargie et où on trouve des caractéristiques dimensionnelles d'organisation et de fonctionnement relevant des conditions particulières des fermes. Dans ces zones dont on parle, on retrouve au début du siècle les familles les plus nombreuses du pays; la taille des familles agricoles variait entre 9 à 12 personnes en Toscane et en Emilie-Romanie, 18 à 20 en Marche, et même près de 30 dans le Veneto. On pouvait voir une unité productive avec des divisions internes du travail et une structure de l'autorité bien définie. Nonobstant l'évolution des bases économiques au cours du temps, de telles familles étaient organisées d'une telle façon qu'elles avaient un certain contrôle sur leur propre destin, et une autonomie 
basée sur l'expérience de leur capacité d'organisation, expérience suffisamment stable pour se renforcer avec le temps (Bagnasco, 1988).

Après la seconde guerre, la famille paysanne évolue dans diverses directions, mais se maintient souvent comme un centre d'unité des fonctions et des décisions économiques et sociales. Dans les familles à prépondérance agricole, la division du travail entre les personnes se structure en fonction de l'activité agricole, du travail externe qui permet l'apport d'autres revenus et des activités de services internes à la famille. Lorsqu'on passe à une activité industrielle complémentaire, celle-ci continue dans plusieurs cas à être reliée économiquement et sociologiquement à l'agriculture, comme par exemple posséder et cultiver un potager, élever des animaux de basse-cour, ou demeurer au village et travailler à la ville. Ce que montrent les résultats d'une recherche dans la région de Marche où on montre que des $45 \%$ des familles qui se limitent au travail à la ferme, $39 \%$ possèdent et cultivent un potager, $20 \%$ ont directement contribué à la construction de leur propre maison, $20 \%$ élèvent des animaux de basse-cour et l'autre $50 \%$ produisent de la marmelade, du vin, etc. (Paci, 1980).

La «famille villageoise modifiée» (Bagnasco, 1988) selon diverses formes, assure une offre de travail peu coûteuse mise au service de la maison, à la production pour l'autoconsommation, à l'apport de revenus divers gérés conjointement, ce qui signifie un abaissement significatif des coûts de productions et une souplesse certaine, puisque la famille peut offrir toutes sortes de besognes à un membre temporairement sans travail. Finalement, ce système est très important pour assurer la socialisation des jeunes familles, pour assurer un soutien réciproque et une autorité hiérarchique interne, mais aussi pour partager les prestations et acquisitions ou s'occuper des contestations externes, pour gérer les activités complémentaires et subsidiaires et pour assurer une formation de base artisanale polyvalente (Bagnasco, 1988).

Ce processus de formation, comme on le verra est important aussi pour l'entrepreneur, même s'il demeure toutefois secondaire dans le contexte plus général de cette société à industrialisation diffuse. Plus importants encore sont les autres moyens de formation d'acquisition et de diffusion d'habiletés et de compétences professionnelles, de socialisation industrielle, et surtout, de la formation des entrepreneurs. Ils proviennent en particulier de la ville, des grands établissements industriels ou du développement de tissus industriels artisanaux; ils ont relevé au début du siècle d'initiatives institutionnelles pour la création d'écoles techniques pour artisans et pour les opérateurs professionnels.

De telles ressources localisées dans la ville ont acquis une importance toujours plus grande pour le développement industriel. La circulation continuelle de ces opérations d'une entreprise à l'autre et la forte mobilité de la main-d'oeuvre dépendant du travail autonome sont une caractéristique systématique de ces zones industrielles. Elles ont permis une remarquable diffusion des connaissances techniques et, par conséquent, une possibilité ininterrompue d'expérimentation et d'innovation technologique. 


\section{$4 \quad$ Les ressources de base : l'entrepreneur}

La structure de ces rapports de production dans les campagnes et les caractéristiques de la famille villageoise modifiée semble aussi pouvoir expliquer la dynamique de la formation des entrepreneurs dans les zones à industrialisation diffuse. En effet, comme on l'a vu à la section précédente, la diffusion des connaissances techniques et organisatrices et la socialisation aux valeurs du marché n'est pas une compétence exclusive de milieu urbain. En outre, la forte mobilité, de l'agriculture à l'industrie et du travail dépendant à celui autonome, ne permet pas d'isoler une seule démarche de formation de l'entrepreneur.

Comme on l'a dit après la seconde guerre, la famille villageoise s'est souvent maintenue comme centre unitaire des cohésions et de décisions économiques et sociales. Cette structure, qui a amené quelques uns à créer l'idée de «l'administration familiale» (Paci, 1980), à cause de la qualité semi-entrepreneuriale du chef de famille, est le résultat de l'expérience de métayage et de villageois (dans ce domaine, il y aurait une autre étude à faire qui serait proche de la première).

Le développement de cette qualité entrepreneuriale chez les cultivateurs et les métayers semble être en général, le résultat du rapport difficile entre la «concedente» et la lutte quotidienne pour la subsistance. Une telle qualité, cependant, ne se réalise pas à ces niveaux (l'investissement des capitaux dans le commerce et la transformation dans le sens capitaliste de la production, etc.); elle reste substantiellement une prérogative des «concedente» et des métayers, impliqués par nature dans ce rapport et s'explique par un manque de postes décisionnels. Elle se trouve plutôt dans l'application d'une capacité d'organisation du processus de production relié en grande partie aux exigences de subsistante domestique ou à l'autoconsommation et, en même temps, à une gestion rationnelle de la force du travail familial d'où dépend la possibilité de faire fructifier sa propre part de capitaux pour atteindre peut-être les formes complexes de divisions et de spécialisation du travail (Bagnasco et Pini, 1981). En résumé, donc, les métayers acquièrent les motivations pour développer leurs connaissances techniques (dérivées de l'activité intégrative ou complémentaire au travail agricole), leur capacité d'organisation pour gérer une entreprise, une familiarité avec le marché, tout en ayant des carences pour une plus large vision stratégique.

En effet, deux autres éléments structuraux importants sont nécessaires pour définir le processus de formation des entrepreneurs : la technologie et la ville. D'un côté la ville offre la formation professionnelle, précondition indispensable pour pouvoir se lancer en affaire. Mais un second élément typique de la ville reste important, soit l'insertion dans un réseau des relations techniques et de relations personnelles permettant d'établir des rapports commerciaux ou coopératifs et de répartir le travail nécessaire pour développer l'entreprise.

Ce second élément touche à l'aspect technologique qui permet une liaison «forte» entre les habiletés et les possibilités de «l'auto-emploi». Comme le montrent 
plusieurs recherches, il existe en effet des «filières» de mobilité sociale propre à chaque secteur. Par exemple, dans l'habillement et les équipements, plusieurs entrepreneurs proviennent du commerce, d'artisans dans le bois et le meuble, du secteur de la mécanique ou ateliers d'usinage; à Carpi, l'entrepreneur typique provient ainsi d'une origine entre l'artisan et le commerçant, etc. (Bagnasco et Pini, 1981).

En résumé, lorsque finit cette ascension sociale, on constate que la provenance villageoise de la direction des entreprises des zones d'industrialisation diffuse demeure un phénomène relativement limité; la qualité entrepreneuriale acquise par le métayer peut nécessiter une période de formation professionnelle dans la ville avant la création d'une entreprise industrielle. Et quels que soient les résultats ou le cheminement réel suivi par les entrepreneurs qui se vantent d'une origine villageoise plus ou moins proche, la plupart ont obtenu une expérience intermédiaire dans la ville, directement ou par l'intermédiaire d'un fils ou un neveu étant passé par la ville. On trouve aussi des micro-entreprises tirées directement de l'agriculture dans des secteurs plus traditionnels et à techniques plus simples. Plus l'unité productive est intégrée, plus sa production est stratégique et fait partie d'un système industriel complexe, plus elle est centrée ou localisée sur la ville. Autrement, la localisation de l'unité productive et à prédominance rurale et le passage de villageois à petits entrepreneurs est direct (Paci, 1980; Bagnasco et Trigilia, 1985). On peut évidemment évaluer l'importance de ces différents cheminements par le succès de chaque système industriel local, dans lequel la majorité des entrepreneurs utilise sa capacité technique pour organiser son entreprise.

Le passage à la ville s'explique parce que c'est là où se concentrent les diverses capacités, les fonctions commerciales et financières et le support administratif et culturel. C'est dans la ville que, depuis la fin du moyen âge, on retrouve les liaisons avec les marchés internationaux indispensables pour le succès du système productif local.

\section{La sous-culture politique et la régulation du marché}

Jusqu'à présent nous avons vu deux éléments structurels fondamentaux pour la régulation économique des zones à industrialisation diffuse. D'abord, nous avons vu le marché, en particulier le marché du travail, fonctionner; mais ses effets de désintégration et d'action anonyme sont compensés par les relations communautaires de la famille. Pour compléter ce tableau, il faut revenir sur les institutions locales comme facteur de régulation discuté déjà dans l'introduction. Comme les districts industriels anciens (Sabel et Zeitlin, 1985), on doit ajouter l'aspect culturel au processus des créations institutionnelles. En effet, la communauté d'une zone ou 
d'un district présente un système assez homogène de valeurs qui s'expriment en termes d'éthique du travail, de l'activité, de la famille, de la réciprocité et du changement (...) Tout ceci constitue une des conditions préliminaires pour la formation d'un district et une des raisons fondamentales de leur reproduction (...). Ces conditions doivent être présentes au point que leur absence peut même décourager l'entreprise d'être créée ou d'évoluer techniquement (...). En parallèle avec ce système de valeurs, il doit exister un système d'institutions et des règles dans le district soutenant ces valeurs et pouvant les transmettre d'une génération à l'autre (Becattini, 1989 : 113).

Cette caractéristique bien particulière des zones de la «Troisième Italie» et l'existence d'un tel ensemble de valeurs et d'institutions expliquent historiquement la structure et l'articulation de la sous-culture politique territoriale (Triglia, 1981, 1986). Ce concept de sous-culture est plutôt complexe et doit être discuté. Il constitue en quelque sorte un instrument théorique pour analyser sociologiquement, d'une part, la norme intégrée pouvant expliquer un système des valeurs partagées et, d'autre part, le mécanisme de négociations et d'échanges entre les divers groupes intéressés par l'intégration de la société (Touraine, 1975; Trigilia, 1986). Avec un tel instrument, Trigilia a analysé ce qu'on peut appeler une particularité significative rencontrée dans les zones à industrialisation diffuse de l'Italie du Centre, soit la persistance, du début du siècle à nos jours, des orientations politico-culturelles bien précises. Les régions centrales (Toscane, Emilie et Umbrie) ont toujours été des régions «rouges» avec d'abord une présence majoritaire socialiste, puis communiste après l'époque fasciste; les régions nord-orientales (Veneto et Trentino) ont toujours été des «zones blanches» à prédominance d'abord catholique, puis démocrate-chrétienne. Dans l'un ou l'autre cas, le système politique local a foumi une réponse politique décentralisée aux problèmes sociaux émergents, comblant. du moins en partie, le vide institutionnel laissé par l'Etat central (Trigilia, 1986).

La sous-culture territoriale, née de la consolidation de mouvements socialistes et catholique durant les premières décennies du siècle, semble avoir diffusé dans la société locale des changements induits par le marché et l'Etat central; elle a permis de limiter la désintégration sociale et de contenir la prolétarisation, à l'aide de diverses formes locales (Trigilia, 1986). Comme on l'a déjà noté, cette évolution a prise différentes formes. Dans les zones socialistes, on a assisté à de plus fortes tensions sociales; et ceci s'est traduit durant la seconde partie de l'après-guerre en un projet collectif alternatif de développement. Dans les zones catholiques, la défense culturelle des valeurs et de l'ordre social traditionnels a été au contraire plus marquée. Dans l'un ou l'autre cas, «la sous-culture» a contribué à préserver un environnement social traditionnel, a forgé les orientations particulières de l'activité économique et a influencé encore par ce moyen les développements suivants (Trigilia, 1986 : 27).

Après la parenthèse fasciste, la sous-culture a «ressurgi». «Le climat de fortes tensions idéologiques qui a caractérisé les années 50 et l'activité des réseaux institutionnels communistes et catholiques ont été surtout orientés pour répandre ou 
reproduire l'appartenance culturelle d'ensemble. Le parti communiste et l'Eglise ont assumé respectivement une présence de base dans cette phase et ont exercé un contrôle et une coordination des autres institutions d'assistance culturelle, récréative ou économique, liées à cette sous-culture et à ses promesses ou ses supports» (Trigilia, $1986: 31$ ).

Sur le plan local, donc, la sous-culture a favorisé une médiation des intérêts à travers leur influence sur les relations industrielles et sur l'activité du gouvernement local. Elle a contribué à la régularisation économique locale, fournissant les ressources, au travers des pressions du gouvernement central ou régional pour soutenir le développement et faciliter l'accord et l'intégration entre les divers intérêts. L'impact a été moins important, mais non négligeable dans le champ économique, tout en assumant un poids majeur dans la politique territoriale et sociale. Dans cette volonté, la différence entre les deux sous-cultures s'est manifestée récemment, à travers une accentuation de la planification dans les zones rouges et une gestion plus individualiste des rapports dans les blanches.

\section{De la régulation sociale à l'intervention économique}

Le dernier modèle qui décrit trop sommairement les correspondances des phases de décollage et de consolidation, de l'après-guerre aux années 70, n'existe plus probablement; encore moins, ce modèle ayant enraînté attirances et transformations, a peu modifié sensiblement la structure et le fonctionnement de l'économie. Aussi, c'est un nouveau modèle, qui ne semble pas encore bien défini, qui peut expliquer les changements analysés dans cette dernière section.

Dans les années 80, avec leur succès économique, les zones à industrialisation diffuse ont vu apparaître les premières difficultées. Dans certains cas, on assiste à des crises sectorielles; dans d'autres, plus nombreux encore, on voit «des crises de croissance». Dans tous les cas, les districts industriels et les systèmes locaux des petites entreprises doivent se mesurer à de nouveaux défis technologiques et compétitifs. Le point de départ de cette nouvelle situation se trouve dans l'intégration majeure de l'économie locale aux filières technologiques et aux marchés internationaux. Dans la plupart de ces cas, cette évolution est directement à la base du succès économique de la zone, conjuguée avec une évolution endogène de la société locale jusqu'au «bouleversement» entraînant un processus de tertiarisation, la scolarisation des masses et la diffusion d'orientations et de comportements post-industriels.

D'un côté, cette situation met directement en cause les règles institutionnelles de régulation d'une croissance relativement spontanée ayant pour but d'élaborer des politiques et instruments d'interventions actives pour soutenir le développement 
économique. En d'autres termes, on doit se poser la question si on peut seulement activer et valoriser les ressources techniques, les connaissances et les matériaux déjà présents dans la zone ou plutôt créer ou acquérir à l'extérieur les ressources nécessaires pour soutenir la concurrence internationale, soient les techniques microélectroniques des services avancés aux entreprises et la capacité d'organisation des instruments pour la pénétration des marchés extérieurs. De plus, le maintien des spécialisations de production et des connaissances complexes du système productif local a aussi réduit le rôle des «purs entrepreneurs». Les nouveaux segments de production se trouvent directement insérés dans la compétition internationale et doivent recourir à l'autonomie des hommes et aux techniques et structures adaptées pour soutenir les nouveaux défis. Ces changements de perspective et ces nouvelles exigences se traduisent plus d'une fois en une situation objective difficile, que l'entreprise doit péniblement affronter. Par conséquent, on se trouve devant la nécessité pour les entreprises, d'élaborer des formes de coopération plus complexes et, pour le système local, de recourir à l'intervention politico-institutionnelle.

D'un autre côté, cette solidité et fonctionalité de la sous-culture politique vient remettre en cause l'actualité et la croissance de la différenciation des intérêts à l'intérieur de la société locale. Dans certains cas de nouveaux entrepreneurs «exportent le marché», et surtout les groupes sociaux se reconnaissent moins dans la société locale. Les jeunes, en particulier, posent encore plus de questions. Capecchi (1987) souligne comment ils deviennent étrangers au projet collectif et à ce processus favorisant l'émergence de l'entrepreneur à l'encontre des générations précédentes. Dans les autres zones, les jeunes semblent préférer un travail stable de banquier à la succession de leur père dans l'entreprise (Baptiste et Michelsons, 1988). Plus souvent encore, toutefois, les jeunes semblent se diriger vers les activités tertiaires ou les nouvelles technologies, favorisant ainsi le développement de la zone. D'autres formes d'évolution des intérêts peuvent conduire à la cassure - plaît à Dieu qu'elle soit temporaire - de la société locale; ce qui demande de nouvelles formes de médiation entre les divers intérêts. L'histoire récente de Valdesa, déjà mentionnée plus haut (Michelsons, 1989; Baptiste et Michelsons, 1988), aussi évidente que limpide est un cas où il y a un problème de création de nouvelles ressources et un problème de médiation entre les intérêts qui confrontent les institutions locales. Naturellement, d'autres cas, avec d'autres dynamismes spécifiques soutiennent ces affirmations.

L'évolution, encore récente, de la sous-culture et de ses institutions, de la famille et de la communauté, à la base du développement et du marché de la société locale, devient comme une barrière entre les individus et les rapports de production et entre l'entreprise et le marché. Le modèle «classique» des zones à industrialisation diffuse était basé sur la famille élargie, les cycles occupationnels et de revenus de ses membres et l'offre des ressources pour le développement de la petite entreprise. Dans ce modèle, les institutions fournissaient le crédit, les services sociaux, les facilités économiques et la médiation entre des intérêts pas trop divergents; on obtenait l'adhésion dans le contexte d'une société qui se reconnaissait dans un système de 
valeurs partagées et y trouvait les avantages globaux de ce développement. Les changements en cours grugent les composantes culturelles et institutionnelles traditionnelles et sollicitent de nouvelles formes de règlements. On peut y voir «un problème de construction politique de nouvelles conditions pour la poursuite du développement» (Trigilia, 1986:37). Le développement économique dépend de la mesure croissante des prestations politiques efficaces, tant dans la création de ressources que dans la médiation des intérêts. Le consentement devient ici plus instrumental, plus directement relié à la capacité du système politique local à satisfaire les demandes spécifiques. Actuellement, comme on l'a dit, les attentes et les comportementsévoluent, pendant que les règleset les classes sociales ne sont plus stables ou ne font que passer. Par exemple, selon une étude récente, des compétences plus grandes sont aujourd'hui nécessaires pour gérer une entreprise, et, dans plusieurs zones, les canaux de mobilité sociale augmentent et le travail lié à l'autonomie semble se réduire.Mais aussi les ajustements institutionnels locaux doivent être redéfinis. En particulier, l'identification aux groupes d'intérêts syndicats, associations d'entrepreneurs ou d'industries - devient plus importante et les règles des institutions pour la médiation des intérêts au niveau local acquièrent un poids croissant sur le réseau sous-culturel ou se transforment avec de nouvelles règles montantes (Trigilia, $1986: 35$ )

Ainsi ce processus de développement économique et de modernisation sociale présente de nouveaux défis à cette société d'industrialisation diffuse de l'Italie du Centre. Cette société devient désormais une société complexe qui a connu un modèle de développement et de croissance économique original avec beaucoup de problèmes économiques, culturels et politiques typiques de la société postindustrielle. La nécessité de combiner de façon synergique les intérêts sociaux, les connaissances techniques et les structures économiques devient encore plus urgente que dans le passé. Mais à la différence de ce passé, il n'est plus suffisant de créer ces conditions dans un contexte de libre choix des intérêts individuels, ni de stimuler les ressources locales selon le libre jeu du marché, des formes plus structurées et prospectives des organisations de coopération et l'intervention et la concertation politiques sont devenues indispensables. Les solutions concrètes sur le plan d'organisation comme sur celui institutionnel devront toutefois continuer, comme par le passé, a interpréter et valoriser de façon soutenue les ressources locales, sans chercher à reproduire un modèle abstrait optimal du système économique et social. 


\section{BIBLIOGRAPHIE}

Bagnasco, A., (1977), Tre Italie, Bologne, Il Mulino.

Bagnasco, A., (1985), Lo sviluppo a industrializzazione diffusa, dans A. Michelsons, Tre incognite per lo sviluppo, Milan, Angeli.

Bagnasco, A., (1988), «La costruzione sociale del mercato, Bologne, II Mulino.

Bagnasco, A. et Pini, R., (1981), Sviluppo economico e trasformazioni sociopolitiche dei sistemi territoriali a economia diffusa. Economia e struttura sociale. Quaderni della Fondazione Feltrinelli, 14.

Bagnasco, A. et Trigilia, C., (1984), Societa' e politica nelle aree di piccola impresa. Il caso di Bassano, Venize, Arsenale.

Bagnasco, A. et Trigilia, C., (1985), Societa' e politica nelle aree di piccola impresa. Il caso della Valdelsa, Milan, Angeli.

Baptiste, F. et Michelsons, A., (1988), «Etude du cas de l'Alta Valdelsa» dans Milieux industriels et systèmes industriels locaux, Lyon, Groupe Lyonnais de Sociologie Industrielle.

Becattini, G., (ed.), (1975), Lo sviluppo economico della Toscana, Florence, Irpet.

Becattini, G., (1978), «The development of light industry in Tuscany», Economic Notes, 2-3, Sienne.

Becattini, G., (1989), «Riflessioni sul distretto industriale marshalliano come concetto socioeconomico", Stato e mercato, 25.

Bianco, L. et Luciano, A., (1982), La sindrome di Archimede, Bologne, Il Mulino.

Brusco, S., (1975), «Economie di scala e livello tecnologico nelle piccole imprese», dans A. Graziani, (ed), Crisi e ristrutturazione nell' economia italiana, Turin, Einaudi.

Brusco, S., (1982), «The Emilian model : productive decentralization and social integration», Cambridge Journal of Economics, 6.

Capecchi, V., (1985), «Tendenze dell' artigianato» dans AA.,VV., Problemi dello sviluppo industriale e dell' occupazione a Bologna, Bologne, Il Mulino.

Capecchi, V., (1987), «Les transformations du petit entreprenariat dans une région de l'Italie», Annales de Vaucresson, 26.

Capecchi, V., Barbagli, M. et Cobalti, A., (1988), La mobilita' sociale in Emilia Romagna, Bologne, Il Mulino.

Fuà, G. et Zacchia, C., (1983), Industrializzazione senza fratture, Bologne, Il Mulino.

Ganne, B., (1989) «Industrialisation diffuse et systèmes industriels localisés», Lyon, Groupe Lyonnais de Sociologie Industrielle.

Michelsons, A., (1983), «La crisi del Fordismo e le possibilita' di sviluppo di un'industria a specializzazione flessibile a Torino", Annali della Fondazione Einaudi, XVll.

Michelsons, A., (1985), «La problematica dell'industrializzazione diffusa nelle scienze sociali italiane» dans R. Innocenti, (ed.), Piccola citta' e piccola impresa, Milan, Angeli.

Michelsons, A., (1986), «Turin between Fordism and flexible specialization. industrial structure and social change 1970-85», Thèse de doctorat, Université de Cambridge.

Michelsons, A., (1989), «The case of Fiat Auto and Olivetti», à paraître dans Economy \& Society.

Paci, M., (ed.), (1980), Famiglia e mercato del lavoro in un' economia periferica, Milan, Angeli.

Piore, M. et Sabel, C., (1984), The Second Industrial Divide, New York : Basic Books.

Sabel, C., (1986), «Industrializzazione del Terzo Mondo e nuovi modelli produttivi», Stato e mercato, 17. 
Sabel, C. et Zeitlin, J., (1985), «Historical alternatives to mass production», Past \& present, 108.

Saglio, D., (1989), «La construction sociale des marchés», Lyon : Groupe Lyonnais de Sociologie Industrielle.

Scamuzzi, S., (ed.), (1987), Modernizzazione ed eterogeneita' sociale. Il caso piemontese, Milan, Angeli.

Touraine, A., (1975), La produzione della societa', traduction, Bologne, Il Mulino.

Trigilia, C., (1981), «Sviluppo economico e trasformazioni sociopolitiche dei sistemi territoriali a economia diffusa. Le subculture politiche territoriali», Quaderni della Fondazione Feltrinelli, 16.

Trigilia, C., (1986), Grandi partiti e piccole imprese, Bologne, Il Mulino. 